\title{
Willingness to Pay for Air Quality Improvements in Klang Valley Malaysia
}

\author{
${ }^{1}$ Rafia Afroz, ${ }^{1}$ Mohd Nasir Hassan, ${ }^{1}$ Muhamad Awang and ${ }^{2}$ Noor Akma Ibrahim \\ ${ }^{1}$ Department of Environmental Sciences, Faculty of Science and Environmental Studies \\ University Putra Malaysia, 43400 UPM, Serdang Selangor Darul Ehsan Malaysia \\ ${ }^{2}$ Institute for Mathematical Research, University Putra Malaysia
}

\begin{abstract}
This research employed contingent valuation method (CVM) to estimate the willingness to pay (WTP) of the respondents to improve the air quality in Klang Valley. The samples were divided according to different question formats i.e. open ended(OE), dichotomous choice(DC) and payment $\operatorname{card}(\mathrm{PC})$. The objective of this study is to investigate the convergent validity of contingent valuation estimates by comparing the WTP values of different question formats. The comparison of responses to different question formats in terms of positive response rate, valid zero, rejection of contingent market was carried out using the z-tests. Paired t-tests of equivalent means were also conducted to test the equality of the mean value of the WTP of the respondents for different question formats. The results of the study suggested that the WTP values of the respondents do not differ significantly across different question formats and the WTP values of the respondents using the DC format were the highest. However due to the nature of the question format, the mean WTP values using the DC method would normally be higher than those of OE and PC. The aggregate WTP value of the respondents was RM0.91 billion for air quality improvement in Klang Valley.
\end{abstract}

Key words: Contingent valuation method, willingness to pay, open ended, dichotomous choice

\section{INTRODUCTION}

In Malaysia the ambient atmospheric conditions have progressively deteriorated due to urbanization and industrial development.Transport vehicles and industrial emissions are the major sources of pollutants in the Klang Valley atmosphere, a problem that has been aggravated by the tremendous increase in the number of mobile sources. In Klang Valley, the concentration of $\mathrm{SO}_{2}, \mathrm{NO}_{2}$ and $\mathrm{CO}$ is always under the Malaysian air quality standard. But the concentration of $\mathrm{PM}_{10}$ exceeds the Malaysian air quality guideline in this area (1). So, there is need to evaluate the air quality improvement in Klang Valley, Malaysia. A common problem when dealing with this type of issue is to obtain a monetary value for a good that is intangible and does not have a market price. The increasing importance given to the valuation of intangible good during last decades has given rise to the development of several valuation methods [2]. One of these is the contingent valuation method. This method presents consumers with hypothetical opportunities to buy public goods, thus circumventing the absence of a real market for them. The resulting information is very useful to the decision makers since it records both the direction and the strength of a respondent's preferences [3]. In contingent valuation studies, it was found that the WTP values of the respondents varies across different question formats and therefore, one is confused about the validity of contingent valuation estimates. Many studies compare the WTP values of different question formats to test the convergent validity of the contingent valuation estimates. While many researchers $[4,5]$ have compared WTP of the respondents by positive response rate, this study looks at additional comparisons. The comparison of positive response rate is certainly important and this is mostly reported in the literature, but comparisons of other responses such as valid zero WTP and rejection of contingent market are equally important when comparing the values given by the respondents using different question formats. This study investigates the convergent validity of contingent valuation estimates by comparing the WTP values of different question formats. The comparison of responses to different question formats in terms of positive response rate, valid zero and rejection of contingent market was carried out using the z-tests. The second major issue considered is the distribution of WTP values across different question formats. The mean value of the WTP distribution has been emphasized by the researchers to find the reliability and validity of contingent valuation measures [6-8]. In this study, paired t-tests of equivalent means were also conducted to test the equality of the mean value of the WTP of the respondents for different question formats.

Theoretical Framework of Contingent Valuation Method for Air Quality Improvement: The

Corresponding Author: Rafia Afroz, Department of Environmental Sciences, Faculty of Science and Environmental Studies, University Putra Malaysia, 43400 UPM, Serdang. Selangor Darul Ehsan Malaysia 
contingent valuation method is a hypothetical survey based method that asks the respondents to place the value of non-market goods. The problem of the respondents in this framework will be to maximize their utility level by choosing the most preferred combination between market and non-market goods and services, subject to certain constraints imposed by the income level and the price of the goods. The utility of the respondents depends on their income, socioeconomic characteristics, consumption of market goods and non-market goods. Here, the non-market good is air quality improvement in Klang Valley. So, the utility function of the respondents can be written as follows:

$$
V=U(Y, S, X, Q)
$$

Where, $V$ is utility of the respondents, $Y$ is income, $S$ is a vector of the socio-economic characteristics of the individual, $X$ is market goods and $Q$ isair quality improvement in Klang Valley. Now, because the utility function is increasing in all its argument, an improvement or increase in the level of provision of any particular non-market good will leave the respondents at a higher level of utility. If an air quality management program improves the air quality in Klang Valley from its current state $\left(Q_{0}\right)$ to its original state $\left(Q_{l}\right)$, the utility function is as follows:

$$
V\left(Y-W T P, S, X, Q_{1}\right)=V\left(Y, S, X, Q_{0}\right)
$$

$$
\operatorname{Pr} o b\{y e s\}=\operatorname{Pr} o b(A \leq W T P)=1-G_{W T P}(A)
$$

An equivalent way of defining the probability of acceptance is using the following equation:

$$
\operatorname{Pr} o b\{y e s\}=\operatorname{Pr} o b\left\{V\left(Y-A, S, X, Q_{1}\right\}+\varepsilon_{1} \geq V\left(Y, S, X, Q_{0}\right)+\varepsilon_{0}\right.
$$

Defining $\Delta v=V\left(Y-A, S, Q_{1}\right)-V\left(Y, S, Q_{0}\right) \quad$ and $\eta=\varepsilon_{1}-\varepsilon_{0}$ with $F \eta($.$) denoting the cumulative$ distribution function of $\eta$, the probability of acceptance can be written as follows:

$$
\operatorname{Pr}(\text { yes })=F \eta(\Delta V)
$$

\section{MATERIALS AND METHODS}

Survey Design and Sampling Method: The on-site survey was employed in the five urban areas of Klang Valley i.e. Shah Alam, Kajang, Klang, Gombak, KualaLumpur and Petaling Jaya. Two thousand seventy nine samples were selected randomly from these five urban
Where WTP is the amount a respondent would be willing to pay to secure a welfare gain resulting from improving air quality to its original, i.e. the change from $Q_{0}$ to $Q_{l}$. This amount corresponds to the Hicksian compensation variation for the proposed change. Now, following the seminal article by [10] if we assume that utility function has some components which are unobservable to the researcher and are treated as stochastic, then the respondent's utility can be written as follows:

$$
V(Y, S, X, Q)=U(Y, S, X, Q)+\varepsilon
$$

Where $\varepsilon$ is a random distribution term with an expected value of zero. If the respondent is asked to pay the amount of money $\mathrm{A}$ as the increase in fuel price for a change in $Q\left(Q_{0} \longrightarrow Q_{1}\right)$, the respondent will accept the offer if

$$
V\left(Y-A, S, Q_{1}\right)+\varepsilon_{1} \geq V\left(Y, S, Q_{0}\right)+\varepsilon_{0}
$$

Where $\varepsilon_{0}$ and $\varepsilon_{1}$ are identically and independently distributed random variables with zero means. The respondent's response is a random variable that will have some cumulative distribution $G_{W T P}(A)$. Therefore, the probability that a respondent will accept the suggested increase in fuel price A can be written as follows:

areas in Klang Valley ( 400 samples from Shah Alam, 430 samples from Kajang, 420 samples from Klang, 410 samples from Kuala-Lumpur and 419 samples from Petaling Jaya). The respondents were randomly selected who agreed to participate in the survey. In case of many family members, one person was chosen for the survey. However, a self-administered questionnaire was given to those who preferred to complete the questionnaire by themselves. All the respondents are 18 years old and above. Before the final survey a pretest was conducted in 23 November, 2001. The first pretest was ascertained in 20 interviews that the respondents did understand the question asked. About 50 people were interviewed in the second pretest after one month, which focused on the range of the bids used in the WTP questions. After modifying the questionnaire, the final survey was conducted in January, 2002. The whole sample was divided into three split samples according to the following question formats: open ended, dichotomous choice and payment card format. In the dichotomous choice format, respondents were confronted with only one single bid and therefore a further subdivision of samples is necessary. In the payment card method a respondent has to value all bids and therefore there is no need to subdivide these samples further. Open-ended willingness to pay values in the pretest ranged from 0 
sen to 50 sen. Since the pretest involved a small number of responses and since some researchers have had problems with a number of respondents saying yes to their highest bid amount [7], the range was widened for the payment card and open ended formats. Payment card values included the following amounts: 1, 3, 5, 10, $15,20,25$, and 50 sen. The money amounts were chosen to approximate a lognormal distribution, the

\begin{tabular}{|c|c|c|c|c|c|}
\hline $\begin{array}{c}\text { Increase } \\
\text { in } \\
\text { Fuel } \\
\text { price } \\
\text { (sen) }\end{array}$ & Yes & $\begin{array}{c}\text { Rather } \\
\text { yes }\end{array}$ & $\begin{array}{l}\text { Don't } \\
\text { know }\end{array}$ & $\begin{array}{c}\text { Rather } \\
\text { yes }\end{array}$ & No \\
\hline 5 & & & & & \\
\hline 10 & & & & & \\
\hline 15 & & & & & \\
\hline 20 & & & & & \\
\hline . & & & & & \\
\hline - & & & & & \\
\hline 50 & & & & & \\
\hline
\end{tabular}

dichotomous values were randomly assigned to survey respondents and included the following money amounts: 1, 5, 10, 15, 20, 25 and 50. The dichotomous choice question was a single bounded question [8, 10$]$.

Questionnaire Design: The questionnaire has three sections. The first section includes questions relating to the knowledge and attitudes of the respondents towards environment and air pollution in general. The second section focused on the valuation questions. Four show cards were presented to the respondents. The show cards (Show cards have been shown in appendix) includes the following topics:

* Major sources of air pollution in Malaysia

* Health effects of air pollution

* Air quality status in the Klang Valley

* The concentration of ambient air quality in Malaysia and the Malaysian air quality guideline.

The air quality management program proposed 20percent reduction in the concentration of $\mathrm{PM}_{10}$ to make the air quality consistent with the Malaysian air quality guideline. The program includes the following measures:

* Strict enforcement of traffic regulation

* The creation of non-traffic areas in some parts of Klang Valley

* Reducing traffic congestion

* The installation of catalytic converters on all cars

* Increase the use of natural gas in the transport sectors
* Decrease the use of gasoline and diesel

* Increase the use of public transportation in urban areas

After describing the air quality management program the respondents were asked how much they were willing to pay to improve the air quality in Klang Valley. The third section includes the socioeconomic characteristics of the respondents.

Air Quality Valuation Questionnaire

Open-Ended Question Format: After describing the air quality status in Klang Valley and air quality management program to improve air quality in Klang Valley, the respondents were asked the following open ended valuation question:

"Obviously the implementation of this program incurs cost, which would be directly or indirectly paid by us. The government will finance this program through an increase in fuel price that will increase your family expenditures. When you consider your household's income and expenditure, are you willing to pay this cost so that the government may achieve this program?"

Respondents who answer yes, are then asked the WTP question:

"How much are you willing to pay? ..............."

Remember that this will give you less money for, for example, food, clothing, shoes, travel car use and savings.

Dichotomous Choice Format: The respondent was asked the following dichotomous valuation question:

"Obviously the implementation of this program incurs cost, which would be directly or indirectly paid by us. The government will finance this program through an increase in fuel price that will increase your family expenditures. When you consider your household's income and expenditure, are you willing to pay this cost so that the government may achieve this program? Remember that this will give you less money for, for example, food, clothing, shoes, travel car use and savings."

Respondents who answer yes, are then asked the WTP question:

"Are you willing to pay .... Sen increase in fuel price that the government may achieve this program?"

The respondents were randomly divided into the bid level, which was taken from pilot survey.

Payment Card Format: As in dichotomous choice version, the respondents were asked whether they support the program at all. Respondents who answer yes, are then asked the WTP question:

"Are you willing to pay an increase in fuel price that the government may achieve this program?" 
The respondents who reported a WTP value greater than zero were treated as positive WTP. The respondents who reported a zero WTP were asked a follow-up question to establish their reasons for not wanting to pay. The respondents who chose to answer 'can't afford to pay anything and Ill-health episode is not bad enough' were treated as valid zero WTP. The respondents who chose to answer 'can't say how much avoiding ill-health episode is worth, paying to avoid illhealth is unrealistic, and not used to making decisions like this' were treated as rejection of contingent market.

there are a large number of zero values, Tobit model has been applied. For the PC format, where WTP values are elicited in the form of intervals rather that point estimates, a maximum bounded likelihood model is applied $[5,11]$.

\section{Estimation Method for Dichotomous Question Format \\ Parametric Approach}

Logistic Regression: For calculation of expected WTP, assumption about the distribution of the random variable $\eta$ and the functional form of the difference in indirect utility $\Delta v$ are necessary. Assuming a logistic distribution of the random variable $\eta$ and a linear indirect utility function $v$ in income $y$, the probability of a yes answer can be written as follows:

$$
\operatorname{Pr}(\text { yes })=F \eta(\Delta V)=\left(1+e^{-\Delta V}\right)^{-1}=\left(1+e^{-\alpha-\beta A}\right)
$$

where,

$$
\Delta V=V_{1}-V_{0}=\alpha_{1}+\beta(y-A)-\alpha_{0}-\beta y=\alpha-\beta A, \alpha=\alpha_{1}-\alpha_{0}
$$

is a constant and $\beta$ the marginal utility of income.

For simplicity the household characteristics are omitted. With increasing fuel price A the utility difference and therefore the probability of a yes answer decrease. It can be seen that the fitting of the discrete response model can be interpreted as estimating the parameters of the distribution function $\mathrm{G}_{\mathrm{WTP}}(\mathrm{A})$ itself. Therefore, the probability of acceptance can be written as follows:

$$
\operatorname{Pr}(\text { yes })=F \eta(\Delta(A))=1-G_{W Y P}(A)
$$

And it makes no difference whether we assume a distribution for $\eta$ or whether we assume a distribution for WTP directly. Note that the linear specification of the utility function of equation, income effects do not appear. Therefore, often a logarithm specification of the utility difference model is used where income is included, though [10] showed that a logarithm specification is not strictly compatible with the utility difference model. However, empirical studies show that
Econometric Analysis: This section focuses on the estimation methods we are using for the three question formats. Whereas open ended (OE) and payment card (PC) methods elicit maximum willingness to pay (WTP) more or less directly, discrete contingent valuation surveys like the dichotomous choice (DC) format only obtain indicator instead of a direct measure of maximum WTP. Therefore, in order to obtain WTP values for DC format a statistical model has to be introduced that links the contingent valuation responses to the price (bid), which respondents faced in the survey. For OE format, where WTP values are not normally distributed, they are truncated at zero and a logarithm specification outperforms the linear logit model derived from the utility difference model. A possible specification where income effects occur and which is compatible with the utility difference model can be written as follows:

$V(q, y ; s)=\alpha_{2}+\beta \ln y ;$ with $\mathrm{j}=0,1$

The difference in utility is then:

$$
\Delta V=\alpha+\beta \ln (y-A)-\beta \ln y\left(1-\frac{A}{Y}\right)=\alpha-\beta \frac{A}{Y}
$$

with $\alpha=\alpha_{1}-\alpha_{0}$ [10]. For the linear utility model of the dichotomous format, mean WTP is given by integrating the logitive function from zero to infinity in the following form:

$\mathrm{E}(\mathrm{WTP})=\int_{0}^{\alpha} F \eta(\Delta y(A)) d A=\int_{0}^{0}\left(1-G_{W T}(A)\right) d A=-\left(\frac{1}{\beta}\right) \ln \left(=e^{\alpha}\right)$

It is assumed that WTP can take only non-negative values, which seems appropriate in the case of air quality improvement.

\section{Estimation Method for Payment Card Question Format}

Parametric Approach: The statistical analysis of payment card data is relatively straightforward. The approach that was followed in this study was outlined in [5], which involves directly estimating the parameters of a willingness to pay function using maximum likelihood techniques. The function states that the individual's contribution to the overall likelihood function is the probability that $W T P_{\mathrm{i}}$ lies between two money amounts on the payment card, conditional on a vector of explanatory variables $X_{i}$ and a set of unobservable factors that are captured by the error term, $\varepsilon_{i}$. More formally, let the vector $t=\left(t_{1}, t_{2}, \ldots . t_{i}\right)$ represents the values on the payment card and let $t_{i}$ represent the value selected by the ith 
respondent. Assume that the willingness to pay function can be written as follows:

$$
\log W T P_{i}=X_{i}^{\prime} \beta+\varepsilon
$$

Where, $\ln W T P$ is the natured logarithm of WTP, $X^{\prime}$ is a vector of explanatory variables such as socioeconomic variables, age, sex, race, marital status and health variables such as duration of ill health episodes, number of symptoms and smoking cigarettes, $\beta$ is a parameter vector, $\mu$ is an error term which is independently normally distributed with mean zero and standard deviation $\sigma$ and However, by simply setting the expected WTP values equal to the internal midpoints, biased WTP values may result. Therefore, we use a multiple bounded likelihood model where WTP becomes a random variable [11]. The probability that a respondent will vote yes, can be written as follows:

$$
\operatorname{Pr}\left(t_{i}\right)=\Phi\left(\frac{\log t_{i+1}-X_{i} \beta}{\sigma}\right)-\Phi\left(\frac{\log t_{i}-X_{i} \beta}{\sigma}\right)
$$

Where $\Phi($.$) is the cumulative density function of the$ standard normal distribution and $t_{i+1}=+\propto$. The log likelihood function can be written as follows:

$$
\log L=\sum \log \left(\Phi\left(\frac{\log t_{i+1}-X_{i} \beta}{\sigma}\right)-\Phi\left(\frac{\log t_{i}-X \beta_{i}}{\sigma}\right)\right)
$$

Where $\sigma$ and the element of $\beta$ have been chosen to maximize the value of this function. By using the estimated values of $\beta$ and $\sigma$ we can calculate values of $L n W T P$. The conditional mean of the $L n W T P$ for any given vector of variables will be $\beta X$ and the mean of the untransformed WTP variable is $\exp \left(\beta X+\sigma^{2} / 2\right)$

\section{Estimation Method for Open Ended Question Format \\ Parametric Approach}

Tobit Regression Model: A Tobit regression model was used in this case because the WTP values were not normally distributed. In this case, if $W T P^{*}=\beta X+\varepsilon$ where $W T P^{*}$ is a latent variable with $\mathcal{E} \sim N\left[0, \sigma^{2}\right]$, the observed variable WTP is censored with respect to

$W T P^{*}$ such that

$$
W T P=W T P^{*} \text { if } W T P^{*}>0
$$

$$
W T P=0 \quad \text { if } W T P \leq 0
$$

where $\mathrm{X}$ denotes the matrix of explanatory variables and the error term $\varepsilon$ is normally distributed with mean zero and standard deviation $\sigma$. The estimate for the regression coefficient $\alpha$ and $\beta$ are obtained through maximum likelihood (ML) techniques. These estimates cannot be compared straightforwardly with the OLS estimates.

\section{RESULTS AND DISCUSSION}

Response Rate of Respondents by Different Question Formats: Table 1 summarizes the WTP values of the respondents according to positive WTP, valid zero and rejection of contingent market across different question formats for the whole survey samples. Results in Table 1 showed that, the largest response with positive WTP was by DC format i.e. 33.86 percent. This is followed by OE format i.e. 15.47 percent. The percentage of the respondents with positive WTP for PC was 13.64 percent. The possible reason for the higher positive response rate for $\mathrm{DC}$ method is that in this method, it was much easier for respondents to answer compared to other two question formats [4]. It was also argued that in the DC method, the respondents are presented with a common and familiar market choice of accepting the bid or not. i.e. purchasing the good at the stated price or not. For OE or PC question formats, the respondents were not given by readily market price to choose and hence the respondents have to search for values, which can be time consuming. Valid zero WTP is almost same in all question formats and rejection in contingent market is higher for the $\mathrm{OE}$ or $\mathrm{PC}$ formats relative to that of $\mathrm{DC}$ format.

Comparison of Positive Response Rates, Valid Zero WTP and Rejection of Contingent Market by Different Question Formats: Table 2 and 3 summarise the comparison of positive response rates, valid zero and rejection of contingent market by different question formats. For the comparison purposes, the Z-tests were carried out to test for values across different question formats. It was hypothesized that the positive response rate, valid zero and rejection of contingent market do not differ across different question formats. Results in Table 2 showed that for positive WTP, the hypothesis of equal proportion has to be rejected because the values using the dichotomous choice format is significantly higher than the values using the $\mathrm{OE}$ and PC question formats. The main reason for this result is that $\mathrm{OE}$ and PC formats are more difficult than the dichotomous choice for the respondents to answer. Results in Table 3 showed that for valid zero answer, the hypothesis of equal proportion was not rejected and 
it could be concluded that there was not significant differences in valid zero answer across different question formats. For rejection of contingent market, the hypothesis of equal proportion has to be rejected and there were significant differences in rejection of contingent market across different question formats. The values using the $\mathrm{OE}$ and $\mathrm{PC}$ formats are significantly higher than the values using the DC question format. Taken together, results from this study, suggested that the dichotomous choice format may ease the valuation task faced by the respondents and this lead to efficiencies in data collection.

Frequency of Bid Amount Across Elicitation Formats: Table 4 shows the frequency of the bid amounts across the elicitation formats. In the open ended question format only three individuals chose the highest offer of $50 \mathrm{Sen}$. In dichotomous choice only ten individuals and in payment card only two individuals chose this amount. A declining percentage of respondents said yes to the dichotomous choice bid amounts as the bids increased.

Dichotomous Choice Format- Parametric Approach: Table 5 summarises the results of the logit models for dichotomous choice method. Valid zero WTP and rejection of contingent market were dropped from the data set as usually done in CVM studies. This study has found that age, marital status, education, income and asthma variables have significant positive effect on the WTP. Other variables do not have any significant effect on the WTP. The positive coefficient on age variable, at level 5 percent level of significance, indicates that holding all other variables constant, older people are more willing to pay than younger people. This is unexpected since increasing age should have a negative effect on willingness to pay. Education and income have a significant positive effect on WTP at 5\% and $1 \%$ level of significance respectively, indicating that, holding all other things constant, educated and higher income people are more willing to pay than less educated and lower income people. This result seems reasonable since a higher level of education and income could be related to a better understanding of the problem. Respondents suffering from asthma have a significant positive effect on WTP of the respondents for improved air quality. For open ended and payment card formats, the signs and significance of the coefficient is almost similar to those of dichotomous choice format.

Open Ended Format- Parametric Approach: Table 6 summaries the results of the tobit models for open ended method. It was found that only marital status, income, education and asthma variables have significant positive effect on WTP. Other variables do not have any significant effect on the WTP values. So, for tobit model sex and age does not have significant effect on WTP for both levels A and B.

Payment Card Format- Parametric Approach: Table 7 summarises the results of parametric models for the payment card method. It was found that sex, age, education, income and asthma variables have significant positive effect on WTP. Other variables do not have any significant effect on WTP values. So, in parametric model marital status does not have any significant effect on WTP.

Comparison of Mean Willingness to Pay of the Respondents by Different Question Formats: Table 8 summarises the mean willingness to pay of the respondents by different question formats. The Pairwise t-tests of equal mean were conducted to compare the mean WTP values across different question formats. It was hypothesized that there was no significant differences in the mean WTP values across different question formats. Results in Table 8 showed that there is no significant difference between the mean WTP values of different question formats. The results of this study lend support to the convergent validity of the contingent market. This result is very similar to the results of the study that had been conducted by Brown et al. [12] has found that there is no significant difference between the WTP values of DC and OE.

\section{Aggregate WTP Value for Air Quality Improvement} in Klang Valley: The WTP values of three question formats have been averaged. The aggregate WTP values have been calculated by multiplying the average WTP by the fuel consumption in year 2000. The average WTP value of the respondents for air quality improvement in Klang Valley is 9.69 Sen fuel price increase per liter. The consumption of fuel in Malaysia is 52.8 billion liters in 2000. Since information on the total number of private vehicle for Klang Valley is not available and the number of total vehicles is the highest in Kuala-Lumpur, in this study the information for Kuala-Lumpur was used. The number of motorcycles and private vehicles is 1.9 million in Kuala-Lumpur and the number of registered vehicles is 10.6 in Malaysia in 2001. So, 10.6 million vehicles consumed 52.8 billion liters in 2000 in Malaysia and the fuel consumption for motorcycles and private vehicles (1.9 million) in KualaLumpur is 9.46 billion litters. So, the aggregate value of the WTP of the respondents for air quality improvement in Klang Valley is $(9.46 \times 9.69)$ or RM0.91 billion for air quality improvement in Klang Valley. 
Table 1: WTP Responses According to Positive WTP, Valid Zero and Rejection of Contingent Market

\begin{tabular}{llll}
\hline Reason & \multicolumn{2}{l}{ Percentage of respondents } & \\
& OE & DC & PC \\
\hline Positive WTP & 15.47 & 33.86 & 13.64 \\
Valid zero WTP & 30.94 & 27.66 & 31.81 \\
Have no extra income but otherwise would contribute & 23.40 & 19.34 & 22.47 \\
Air quality improvement is not important & 7.54 & 8.32 & 9.34 \\
Rejection of contingent market & 53.59 & 38.48 & 43.55 \\
It is the government's responsibility & 26.24 & 15.34 & 22.31 \\
Don't believe that the air pollution mitigating programs would bring the changes & 14.30 & 13.61 & 7.66 \\
The improvement will take place even without individual's contribution & 3.45 & 5.23 & 5.23 \\
It is the responsibility of those who pollute the environment to pay for it & 9.60 & 4.30 & 8.35 \\
\hline
\end{tabular}

Table 2: Comparison of Positive WTP By Different Question Formats

\begin{tabular}{ll}
\hline Question format & Z-test \\
\hline OE,PC & 3.552 \\
PC,DC & 3.762 \\
OE, DC & 3.452 \\
\hline
\end{tabular}

Table 3: Comparison of Valid Zero and Rejection of Contingent Market

\begin{tabular}{llc}
\hline Question format & \multicolumn{2}{c}{ Z-test } \\
\hline & Valid zero & $\begin{array}{l}\text { Rejection of } \\
\text { contingent market }\end{array}$ \\
\hline WTP & 0.023 & 3.234 \\
PC,DC & 0.606 & 3.432 \\
OE,DC & 0.575 & 4.321 \\
\hline
\end{tabular}

Table 4: Frequency of Bid Amount Across Different Question Formats

\begin{tabular}{llll}
\hline $\begin{array}{l}\text { Bid amount } \\
\text { (Sen) }\end{array}$ & OE & DC & PC \\
\hline 1 & 11 & 59 & 15 \\
3 & - & - & 11 \\
5 & 16 & 52 & 33 \\
10 & 31 & 43 & 9 \\
15 & 3 & 37 & 6 \\
20 & 9 & - & 4 \\
25 & 25 & 32 & 15 \\
30 & 4 & - & - \\
35 & 3 & - & - \\
40 & 3 & - & - \\
45 & - & - & - \\
50 & 3 & 10 & 2 \\
\hline
\end{tabular}

Table 5: Logit Model for Dichotomous Choice Format

\begin{tabular}{lll}
\hline Variables & Est. & St. err. \\
\hline Intercept & 3.91 & 1.89 \\
Sex & -0.55 & 0.66 \\
Age & $0.10^{* *}$ & 0.05 \\
Marital status & $1.53^{* *}$ & 0.83 \\
Education & $2.19^{* *}$ & 1.02 \\
Income & $2.29^{*}$ & 0.51 \\
Asthma & $1.36^{*}$ & 0.67 \\
Mean WTP (Sen) & 12.11 &
\end{tabular}

*** Significant at $1 \%$ level of significance and $5 \%$ level of significance
Table 6: Tobit Model Open Ended Question Format

\begin{tabular}{lll}
\hline Variables & Est. & St. err. \\
\hline Intercept & 0.44 & 0.90 \\
Sex & 0.09 & 0.10 \\
Age & 0.02 & 0.02 \\
Marital status & $0.43^{* *}$ & 0.14 \\
Education & $1.15^{* *}$ & 0.34 \\
Income & $0.27^{* *}$ & 0.07 \\
Asthma & $0.20^{* *}$ & 0.09 \\
MeanWTP (Sen) & 9.12 & \\
\hline
\end{tabular}

Table 7: Parametric Model for Payment Card Method

\begin{tabular}{lll}
\hline Variables & Est. & St. err. \\
\hline Intercept & 0.89 & 0.66 \\
Sex & $0.22^{*}$ & 0.01 \\
Age & $0.04^{*}$ & 0.01 \\
Marital status & 0.13 & 0.10 \\
Education & $0.33^{* *}$ & 0.14 \\
Income & $1.26^{* *}$ & 0.39 \\
Asthma & $0.59^{*}$ & 0.15 \\
Mean WTP (Sen) & 9.64 &
\end{tabular}

*,** Significant at $1 \%$ level of significance and $5 \%$ level of significance

Table 8: Comparison of Mean Willingness to Pay by Different Question Formats

\begin{tabular}{lc}
\hline Different question formats & Pair wise t-Tests \\
\hline OE vs PC & 0.507 \\
OE vs DC & 0.903 \\
PC vs DC & 1.234 \\
\hline
\end{tabular}

\section{CONCLUSION}

The study has great implication for the application of contingent valuation method. The results of the study suggested that the WTP values of the respondents do not differ across different question formats Results of the study suggested that the WTP values of the respondents using the DC format were the highest. . However due to the nature of the question format, the mean WTP values using the DC method would normally be higher than those of $\mathrm{OE}$ and PC. The aggregate WTP values of the respondents was RM0.91 billion for air quality improvement in Klang Valley 


\section{REFERENCES}

1. Department of Environment Malaysia (DOE), 1999. Malaysia Environmental Quality Report. Department of Environment, Ministry of Science, Technology and the Environment, Malaysia.

2. Rozan, A., 1999. Contingent valuation of health benefits induced by air pollution: The example of the region of Strasbourg. Ph. D. Thesis. University Louis Pasteur, Strasbourg I.

3. Lockwood, M., P. Tracey and N. Klomp, 1996. Analysing conflict between cultural heritage and nature conservation in the Australian Alps: A CVM approach. J. Environ. Plan. Manag., 39: 357-370.

4. Mitchell, R.C and Carson, R.T. 1989. Using Surveys to Value Public Goods: The Contingent Valuation Method.

Resources for the Future: Washington D.C.

5. Cameron, T.A. and D.D. Huppert, 1989. OLS versus ML estimation of non-market resource values with payment card interval data. J. Environ. Econom. Manag., 17: 230-246.

6. Desvousges, W.H., F.R. Johnson, R.W. Dunford, K.J. Boyle, S.P. Hudson and N. Wilson, 1993. Measuring natural resource damages with contingent valuation: tests of validity and reliability. In Contingent Valuation: A Critical Assessment. (Ed.) Hausman, J.A. Amsterdam, North Holland.
7. Kealy, M.J., M. Montgomery and J.F. Dovidio, 1990. Reliability and predictive validity of contingent values: Does the nature of the good matter? J. Environ. Econom. Manag., 19: 244-63.

8. McFadden, D.L. and G.K. Leonard, 1993. Issues in the Contingent Valuation of Environmental Goods: Methodologies for Data Collection and Analysis. In Contingent Valuation: A Critical Assessment. (Ed.) Hausman, J.A. North-Holland: New York.

9. Hanemann, M.W., 1984. Welfare evaluations in contingent valuation experiments with discrete responses. American J. Agril. Econom., 66: 33241.

10. Bishop, R.C. and T.A. Heberlein, 1979. Measuring values of extra-market goods: Are indirect measures biased? American J. Agril. Econom., 61: 926-30.

11. Welsh, M.P. and G.L. Poe, 1998. Elicitation effects in contingent valuation: Comparison to a multiple bounded discrete choice approach. J. Environ. Econom. Manag., 36: 170-185.

12. Brown, T.C., P.A. Champ, R.C. Bishop and D.W. McCollum, 1996. Which response format reveals the truth about donations to a public good? Land Economics, 72: 152-66. 